\title{
Neuroendocrine Carcinoma of the Gallbladder
}

\author{
Servet Guresci*, Elif Sen, Serra Kayacetin, Gamze Erkilinc, Sezer Kulacoglu
}

Department of Pathology, Numune Education and Research Hospital, Turkey

Received: September 11, 2014; Accepted: September 26, 2014; Published: October 15, 2014

*Corresponding author: Servet Guresci, Department of Pathology, Numune Education and Research Hospital, Turkey, Tel:+90312-508-44 86-4078; Fax: +90312-508-44 85; E-mail: gurescisa@hotmail.com

\begin{abstract}
Neuroendocrine tumors are the rarest tumors of gallbladder. The most agressive variant is neuroendocrine carcinoma that is seen about $0.5 \%$ of all gallbladder carcinomas. We report a case of neuroendocrine carcinoma diagnosed in a 89 year old patient which was diagnosed in a cholesistectomy specimen performed for cholelithiasis.
\end{abstract}

Keywords: Gallbladder; Neuroendocrine carcinoma

\section{To the editor,}

A 89-year-old male presented with a history of right upper quadrant pain. Ultrasonography showed cholelithiasis. Laparoscopic cholecystectomy was performed. On gross examination the gallbladder was $9 \times 4 \mathrm{~cm}$ and the wall thickness was $0.6 \mathrm{~cm}$. The lumen was filled with multiple stones. The mucosa was flattened and tan-white in color. Microscopic examination showed a tumour arranged in sheets infiltrating through the muscle layer with extension to serosa. The tumour cells had round, hyperchromatic nuclei with scant cytoplasm. There were more than 15 mitosis per 10 high power fields. The gallbladder was entirely examined for any type of combined carcinoma and focal high grade dysplasia was demonstrated (Figure 1). Immunohistochemical analysis revealed diffuse immunostaining with CD56, chromogranin A, and pancytokeratin (Figure 2). As a result Neuroendocrine Carcinoma (NEC) of the gallbladder

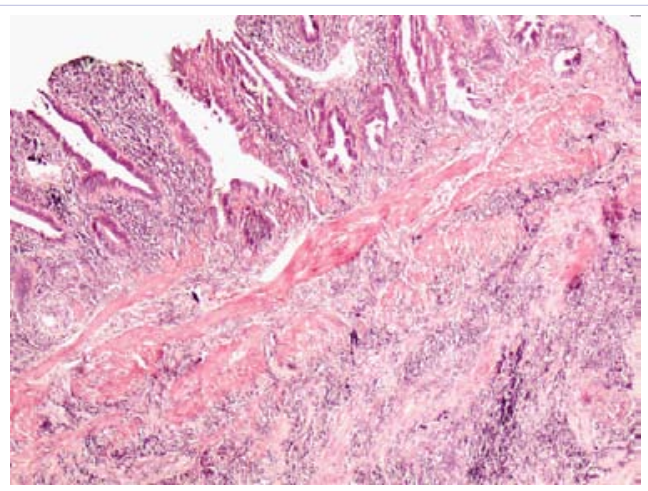

Figure 1: Diffuse infiltration of the tumor through the gallbladder wall, note dysplastic mucosal epithelium (HE x40).

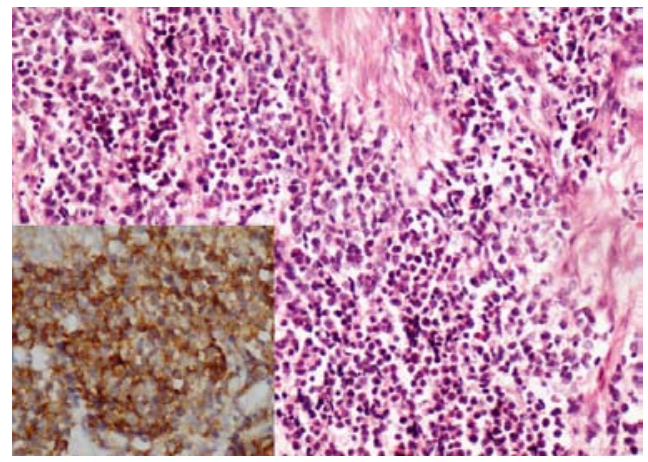

Figure 2: Tumor cells with hyperchromatic nuclei and scant cytoplasm (HE x200) Inset: Diffuse cytoplasmic staining with neuroendocrine marker chromogranin A, (immunoperoxidase $\mathrm{x} 400$ ).

was diagnosed. He received adjuvant chemotherapy with 5 -flurouracil and cisplatin for 2 months but could not tolerate. He died of metastatic disease and complications after 6 months.

Gallbladder carcinoma is the fifth most common gastrointestinal malignancy more than which $90 \%$ is Adenocarcinoma (AC). Neuroendocrine Tumors (NETs) make up of $4 \%$ of all gallbladder cancers and NECs make up even a smaller percentage [1]. The age of presentation ranges from 38 to 81 years and the incidence is higher in females $(1: 1.8)[1,2]$.

Risk factors for NETs include cholelithiasis and chronic inflammation; as most published data describe these, just as ours $[3,4]$. Because gallbladder doesn't contain neuroectodermal cells except than a few in neck region, it's not known for certain where NETs originate from. It is postulated that these tumors arise from multipotent stem cells undergoing gastric and/or intestinal metaplasia with the influence of chronic inflammation [4]. NECs are reported to be pure in $72 \%$ and combined with AC or squamous cell carcinoma in $28 \%$ of the cases, but these states does not have a prognostic significance $[1,2]$. It's imperative to distinguish NECs histologically from poorly differentiated AC, lymphoma and NET to avoid erroneous treatment. Immunohistochemical expression of neuroendocrine markers along with high mitotic activity and co-existing necrosis aid in this problem [5].

Due to lack of systemic symptoms, NECs often present at an advanced stage with only $10 \%$ of cases confined to the 
gallbladder wall at the time of diagnosis, so the overall survival rate is less than $5 \%$ [3]. Currently no rational surgical strategy exists for gallbladder NECs. It seems possible that survival rates be increased by wide surgical resection combined with chemotherapy [4].

\section{References}

1. Bosman TF, Carneiro F, Hruban HR. WHO Classification of Tumors of the Digestive System. 2010; 4th ed. Lyon: International Agency for Research on Cancer; 2010. p. 274-276.

2. Mahipal A, Gupta S. Small cell carcinoma of the gallbladder: report of a case and literature review. Gastrointest Cancer Res. 2011; 4(4): 135136.
3. Nau P, Liu J, Dillhoff M, Forster M, Hazey J, Melvin S. Two cases of small cell carcinoma of the gallbladder. Case Rep Med. 2010;45362. doi: $10.1155 / 2010 / 453624$.

4. Eltawil KM, Gustafsson BI, Kidd M, Modlin IM. Neuroendocrine tumors of the gallbladder: an evaluation and reassessment of management strategy. J Clin Gastroenterol. 2010; 44(10):687-695. doi: 10.1097/ MCG.0b013e3181d7a6d4.

5. Chuang SS, Lin CN, Chu CH, Chen FF. Small cell carcinoma of the gallbladder: report of two cases. Pathol Oncol Res. 1999; 5(3): 235238. 